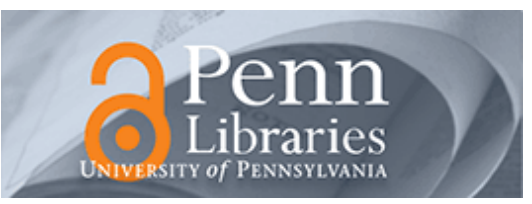

University of Pennsylvania ScholarlyCommons

February 2008

\title{
Real-Time TEM Imaging of the Formation of Crystalline Nanoscale Gaps
}

Douglas R. Strachan

University of Pennsylvania, drstrach@sas.upenn.edu

Danvers E. Johnston

University of Pennsylvania

Beth S. Guitton

University of Pennsylvania

Sujit S. Datta

University of Pennsylvania

Peter K. Davies

University of Pennsylvania, davies@lrsm.upenn.edu

See next page for additional authors

Follow this and additional works at: https://repository.upenn.edu/mse_papers

\section{Recommended Citation}

Strachan, D. R., Johnston, D. E., Guitton, B. S., Datta, S. S., Davies, P. K., Bonnell, D. A., \& Johnson, A. T. (2008). Real-Time TEM Imaging of the Formation of Crystalline Nanoscale Gaps. Retrieved from https://repository.upenn.edu/mse_papers/143

Copyright American Physical Society. Reprinted from Physical Review Letters, Volume 100, Issue 3, Article 056805, February 2008, 4 pages.

Publisher URL: http://dx.doi.org/10.1103/PhysRevLett.100.056805

This paper is posted at ScholarlyCommons. https://repository.upenn.edu/mse_papers/143

For more information, please contact repository@pobox.upenn.edu. 


\title{
Real-Time TEM Imaging of the Formation of Crystalline Nanoscale Gaps
}

\author{
Abstract \\ We present real-time transmission electron microscopy of nanogap formation by feedback controlled \\ electromigration that reveals a remarkable degree of crystalline order. Crystal facets appear during \\ feedback controlled electromigration indicating a layer-by-layer, highly reproducible electromigration \\ process avoiding thermal runaway and melting. These measurements provide insight into the \\ electromigration induced failure mechanism in sub-20 nm size interconnects, indicating that the current \\ density at failure increases as the width decreases to approximately $1 \mathrm{~nm}$.

\section{Keywords} \\ molecular electronic devices, electronic structure of nanoscale materials, electrical properties of metal-to- \\ metal contacts, nanoscale contacts (electronic transpor \\ Comments \\ Copyright American Physical Society. Reprinted from Physical Review Letters, Volume 100, Issue 3, Article \\ 056805, February 2008, 4 pages. \\ Publisher URL: http://dx.doi.org/10.1103/PhysRevLett.100.056805

\section{Author(s)} \\ Douglas R. Strachan, Danvers E. Johnston, Beth S. Guitton, Sujit S. Datta, Peter K. Davies, Dawn A. Bonnell, \\ and Alan T. Johnson
}




\title{
Real-Time TEM Imaging of the Formation of Crystalline Nanoscale Gaps
}

\author{
Douglas R. Strachan, ${ }^{1,2, *}$ Danvers E. Johnston, ${ }^{1}$ Beth S. Guiton, ${ }^{2}$ Sujit S. Datta, ${ }^{1}$ \\ Peter K. Davies, ${ }^{2}$ Dawn A. Bonnell, ${ }^{2}$ and A. T. Charlie Johnson ${ }^{1,2, \dagger}$ \\ ${ }^{1}$ Department of Physics and Astronomy, University of Pennsylvania, Philadelphia, Pennsylvania 19104, USA \\ ${ }^{2}$ Department of Materials Science and Engineering, University of Pennsylvania, Philadelphia, Pennsylvania 19104, USA
}

(Received 9 August 2007; published 7 February 2008)

\begin{abstract}
We present real-time transmission electron microscopy of nanogap formation by feedback controlled electromigration that reveals a remarkable degree of crystalline order. Crystal facets appear during feedback controlled electromigration indicating a layer-by-layer, highly reproducible electromigration process avoiding thermal runaway and melting. These measurements provide insight into the electromigration induced failure mechanism in sub-20 nm size interconnects, indicating that the current density at failure increases as the width decreases to approximately $1 \mathrm{~nm}$.
\end{abstract}

DOI: 10.1103/PhysRevLett.100.056805

Developing molecular-scale electronics requires novel approaches to device fabrication in order to connect macroscopic leads to nanometer scale molecules. One must form highly ordered nanoscale gaps [1] that provide a reproducible environment for the molecule and allow for efficient electrostatic coupling to nearby gate electrodes. Electromigrated nanogaps formed with a single voltage ramp [2] have been suggested for constructing singlemolecule electronics, but the thermal runaway [3], melting [4,5], and stochastic break [2] characteristic of this process do not provide the required reproducibility.

A recently proposed feedback controlled electromigration (FCE) process [6-8] actively adjusts the applied voltage in response to the changing conductance of the weak link, so as to avoid thermal runaway. The feedback system keeps the voltage difference across the nanogap region at the critical level for the onset of electromigration throughout the gap formation process. FCE can be used to form metallic channels with quantized conductance as well as nanoscale gaps [6], and it has been implemented on electron-transparent $\mathrm{Si}_{3} \mathrm{~N}_{4}$ membranes [9] enabling TEM inspection with subnanometer resolution. Images of the resulting nanogaps suggest that they are free of small metal particles that are known to complicate data interpretation, or even dominate electron transport, in molecular electronic devices $[7,10,11]$.

Here we use real-time TEM imaging during the FCE process to monitor the dynamics of electromigration at the subnanometer scale - a scale that was not accessible to earlier investigations of electromigration due to catastrophic thermal runaway [12-14]. We find that welldefined crystal facets appear as the nanogap forms. This is clear evidence that the nanogap does not melt during FCE, in contrast to what can occur during the single voltage ramp procedure [4,5]. We explain the appearance of crystal facets during FCE by an "unzipping" model where electromigration occurs layer by layer, due to preferential thermal excitation of gold atoms that lie on exposed, high-mobility crystal planes of the lattice.

PACS numbers: 85.65.+h, 73.22.-f, 73.40.Jn, 73.63.Rt

Samples are fabricated on freestanding $100 \mathrm{~nm}$ thick, electron-transparent $\mathrm{Si}_{3} \mathrm{~N}_{4}$ membranes supported by $\langle 100\rangle$ silicon wafers [9]. We use photo- and electron-beam lithography with thermal evaporation to define 20 -nm thick gold leads connected by a $50 \mathrm{~nm}$ wide constriction with no adhesion layer. The samples are loaded into a homebuilt holder and imaged in a JEOL 2010F TEM microscope under bright-field conditions [i.e., gold appears dark as in Fig. 1(a)] while FCE is performed. Images throughout include sidegates which are not relevant to the present experiments and can be ignored.

Figures 1(a)-1(e) show several stages in the formation of a nanogap using FCE over a period of about 9 min with the corresponding conductance-voltage curve in Fig. 1(f). (Accompanying videos can be found in the online supporting information [15] with digital time stamps at the upper left of all TEM images.) Figure 1(a) shows a lead that has significantly pinched in from the edges along a slit due to electromigration. At higher resolution in Fig. 1(b), crystal facets and parallel step edges (indicated by the arrow) are apparent along the lower edge where electromigration has begun. This is in contrast to the nonfaceted upper edge of the slit that has not undergone electromigration, possibly due to current crowding effects near the forming nanogap [16]. As FCE is continued in Fig. 1(c) the top edge pinches in, and only at this stage do facets appear, clearly demonstrating that the faceting and edge electromigration mechanisms are closely related. Furthermore, the faceted edges maintain their orientation as FCE proceeds [Figs. 1(b)1(d)] (movie S1 [15]). Thus recrystallization of the nanowire is not occurring, in contrast to previous work [17].

To explain the progressive faceting that appears during FCE, we propose a two-dimensional unzipping model (Fig. 2). Gold atoms on the edge of the narrowed region have the fewest nearest neighbors and are therefore the most weakly bound. As the current is raised, the temperature rises in the nanogap region, and an atom is thermally excited onto the edge as an adatom [Fig. 2(a)]. This metallic adatom will experience an electron wind force [18- 


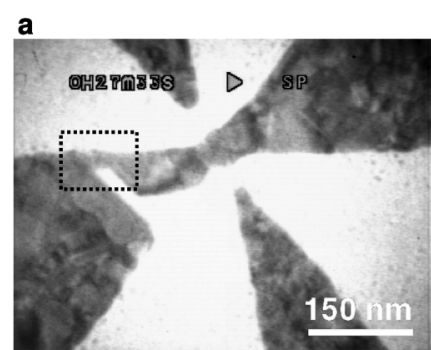

c
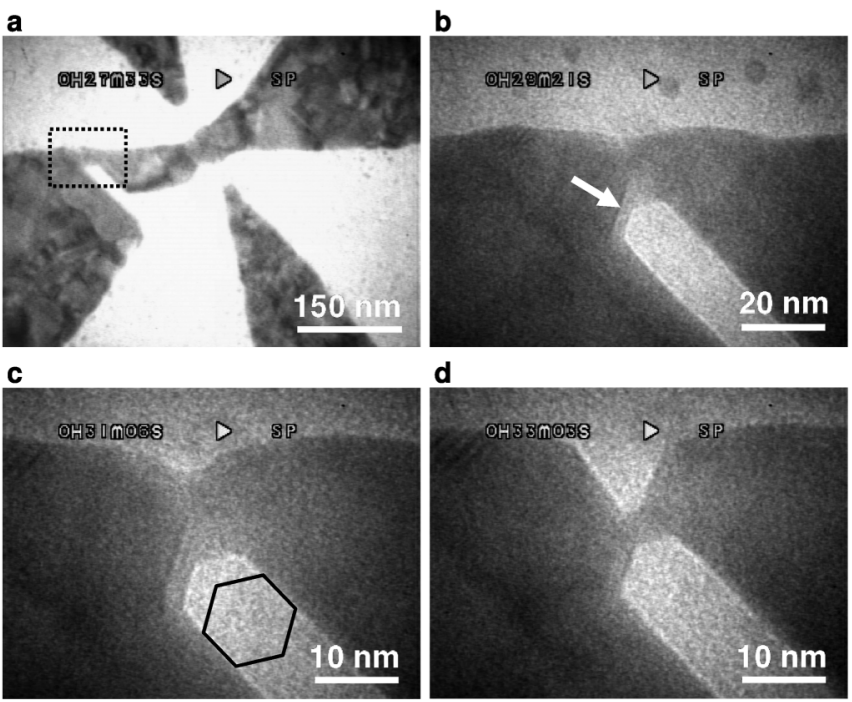

e
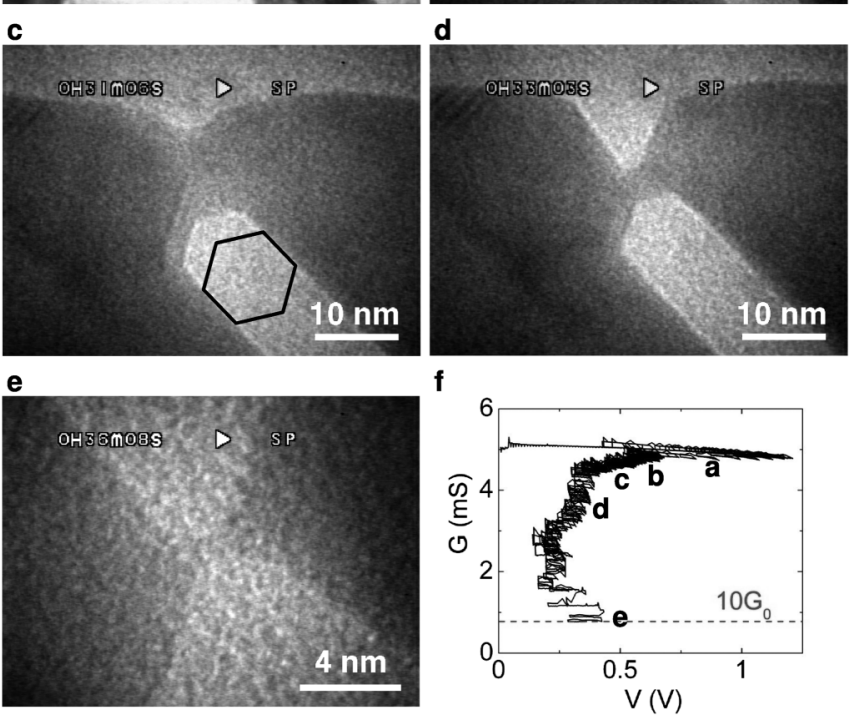

FIG. 1. Formation of a crystalline nanogap. (a) TEM image of a nanogap forming during FCE (gold is dark). Electromigration occurs in the region within the dotted square. (b) Image at a later time showing crystal faceting and thinning in the lower part of the nanogap, where electromigration is occurring. The arrow indicates a step edge. (c) Crystal planes form angles of $120^{\circ}$ in the nanogap region, indicative of electromigration along the $\langle 110\rangle$ directions of $\{111\}$ oriented gold. (d) Electromigration has begun to occur along the top edge, where crystal faceting is now evident. (e) The lead is now pinched down to about $1 \mathrm{~nm}$ in size, corresponding to approximately 10 atomic channels of conduction. (f) The $G-V$ characteristic of the sample with letters denoting the conductance for the corresponding images in the parts (a)-(e) of the figure.

21], and it will be driven along the exposed high-mobility crystal plane by the applied current [Fig. 2(b)]. This process continues with the vacancy driven in the opposite direction towards the corner where the exposed $\{111\}$ crystal planes meet and by the further excitation of lowcoordinated edge atoms [Fig. 2(c)]. This cycle is repeated until the entire layer is removed [Fig. 2(d)]. This unzipping mechanism will lead to a layer-by-layer progression of the faceting which should not depend on whether the adatom or vacancy migration occurs at a greater rate [15]. Although details of the electromigration are complicated as the number of adatoms and vacancies increases during unzipping, it is reasonable to expect that the wind force will continue to drive the atomic motion as the process proceeds [22]. The fact that the angle between facets in Fig. 1(c) is almost exactly $120^{\circ}$ is consistent with electro-
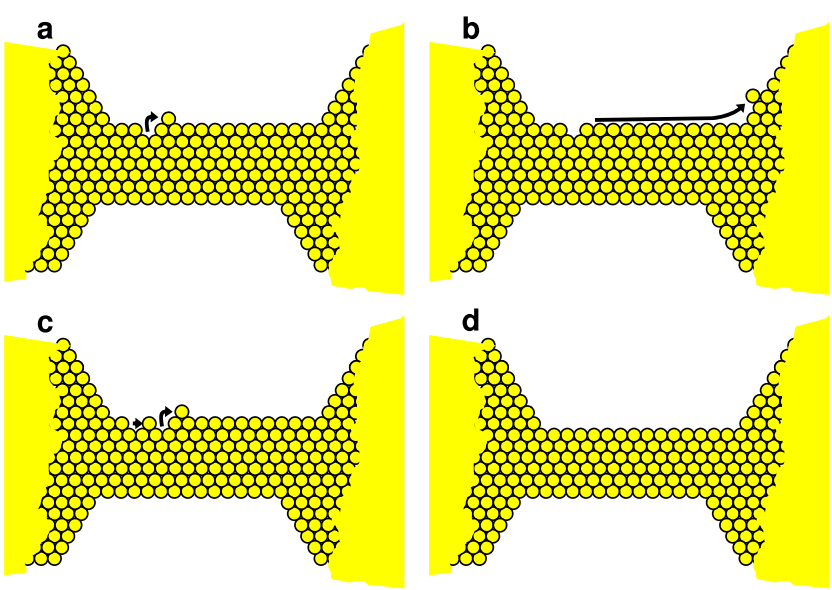

FIG. 2 (color online). Unzipping model of evolution of a nanogap during FCE. (a) Starting with the initial wire, an edge atom is thermally excited. (b) This atom is easily transported away by the applied current along the high-mobility $\langle 110\rangle$ directions. (c) The edge vacancy allows adjacent atoms to easily electromigrate and jump to the edge due to their reduced number of nearest neighbors. (d) The edge is thus unstable once the unzipping of a layer begins.

migration occurring along the close-packed $\langle 110\rangle$ directions of the $\{111\}$ plane within a single gold grain.

FCE can be continued to the point where the bridge is $\sim 1 \mathrm{~nm}$ in diameter [Fig. 1(f), movie S2 [15]]. At this stage, the nanogap region has been narrowed to a single well-ordered continuous bridge with a measured conductance of $\sim 10 G_{0}\left(G_{0}=2 e^{2} / h\right.$ being the conductance quantum) [6]. After a sample has been electromigrated to where the conductance is $\sim 5 G_{0}$, the metallic bridge is unstable even with zero applied current. The nanogap then breaks and evolves within seconds to a gap separation of 3-4 nm, which is likely related to earlier "self-breaking" reports in nanogap formation $[6,9,23]$.

To establish that the gap forms by true electromigration during FCE, we conducted an experiment exploring the asymmetric nature of the gap formation process [9,17,24,25]. As seen in Fig. 3, an initial narrow constriction [Fig. 3(a)] was electromigrated with the applied current such that electrons flow from left to right as indicated in Fig. 3(b). Figures 3(b) and 3(c) show two stages of the initial "forward" FCE, where a faceted nanogap has begun to form at a point on the left ("upstream") side of the narrow constriction (movie S3 [15]). At this point we reverse the electron flow of the partially electromigrated lead [13]. Reversing the current causes the faceted nanogap region to refill with gold, demonstrating that the movement of the gold atoms during FCE is in the same direction as the electron flow [Fig. 3(d), movie S4 [15]]. This process can be continued until the initial void is completely refilled [Fig. 3(e)]. Eventually, a discernible hillock forms at the location of the initial void while a faceted void is apparent on the right side of the narrow constriction [Fig. 3(f), movie S5 [15]]. 
a

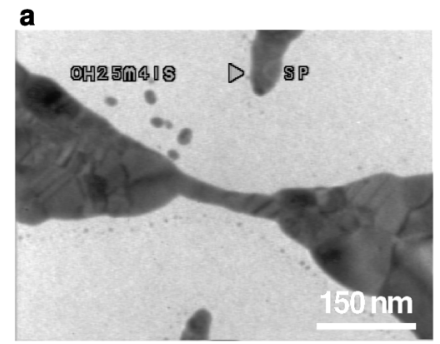

b

c

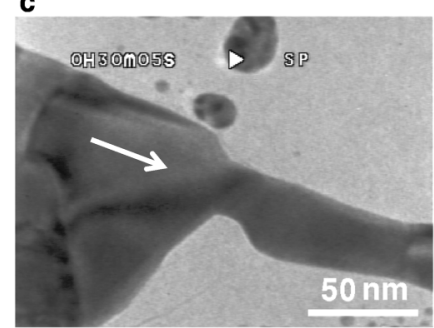

e

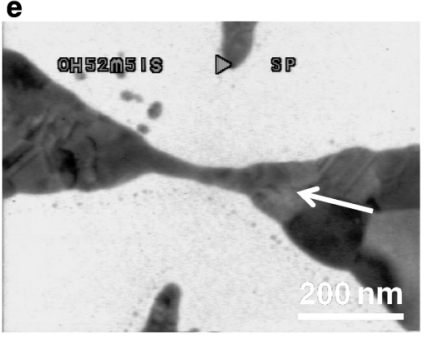

FIG. 3. Reversible electromigration. (a) Initial bowtie structure. (b) Current is applied so that electrons flow from left to right, indicated by the arrow. (c) The lead begins to form a faceted void. Electromigration is ceased at this point when the electromigrated void is clearly the narrowest region of the wire. (d) The applied voltage is reversed and the void begins to fill back in with gold. (e) This is continued to the point where the void is completely filled in. (f) Further electromigration in this direction yields a hillock at the site of the initial void while a faceted void starts to form at the opposite side of the bowtie structure.

The reversibility of FCE demonstrates unequivocally that the atomic motion in this process is due to electromigration and not to thermomigration or sublimation [5]. This also indicates the important role of temperature gradients [26] and surface electromigration during FCE formation of nanogaps. In the initial bowtie geometry [Fig. 3(a)] the localized Joule heating within the narrow bridge causes an increased local temperature, which we estimate to be approximately $530 \mathrm{~K}$ (in rough agreement with other estimates $[4,24,27])$. The temperature rise of the bridge leads to increased gold mobility in that region, and a greater concentration of thermally excited adatoms and vacancies. There is thus a nonzero divergence in the atomic flux at the sides of the bridge due to the electron wind. As a result, gold is depleted from the "upstream side" of the bowtie to form the gap and deposited on the "downstream side" to form a hillock [23] [Fig. 3(f)].

Electromigration failure mechanisms in the sub-20 nm regime have become increasingly important as electronics and interconnects shrink inexorably towards this size and below. Investigating this phenomena is complicated by the exceedingly rapid evolution and thermal runaway that occur when the neck is narrower than about $20 \mathrm{~nm}$ [2837], and by the variation in grain structure for different wires [38]. The current density at failure $J_{F}$ has been reported to both increase [39] and decrease [37] as the wire widths decrease.

Our method eliminates grain structure variability and allows the investigation of size-dependent failure in a single-grain wire as it is narrowed through FCE. Figure 4(a) shows the critical current for electromigration $I^{*}$ (from the sample in Fig. 1) plotted against $W$, the width of the neck at its narrowest point; the data are well fit by the form $I^{*} \propto W^{1 / 2}$ for $W>3 \mathrm{~nm}$. This dependence follows from the assumption that electromigration occurs at a critical power dissipation $P_{n}^{*}=I^{* 2} / G_{n}$ in the neck [6], where $G_{n}$ is the neck conductance. Assuming the neck thickness is a constant $3 \mathrm{~nm}$ in this regime, we expect $G_{n} \propto W$ and $I^{*}=\sqrt{P_{n}^{*} G_{n}} \sim W^{1 / 2}$, in agreement with the data of Fig. 4. This is further supported by the measured $(10 \mathrm{mS})$ conductance when the neck width is $3 \mathrm{~nm}$, which is consistent with a $3 \mathrm{~nm} \times 3 \mathrm{~nm}$ ballistic constriction where each gold atom of diameter $0.28 \mathrm{~nm}$ contributes a conductance quantum. For $W<3 \mathrm{~nm}$, the width and thickness of the neck should decrease together, so one expects $G_{n} \propto W^{2}$ and $I^{*} \propto W$, as seen in Fig. 4, with increased scatter in the data attributed to the crossover to an atomic-sized neck [6]. Overall, the good agreement to $I^{* 2} \sim A$ implies that $J_{F}$ increases as $A^{-1 / 2}$ as the neck narrows for this well-defined

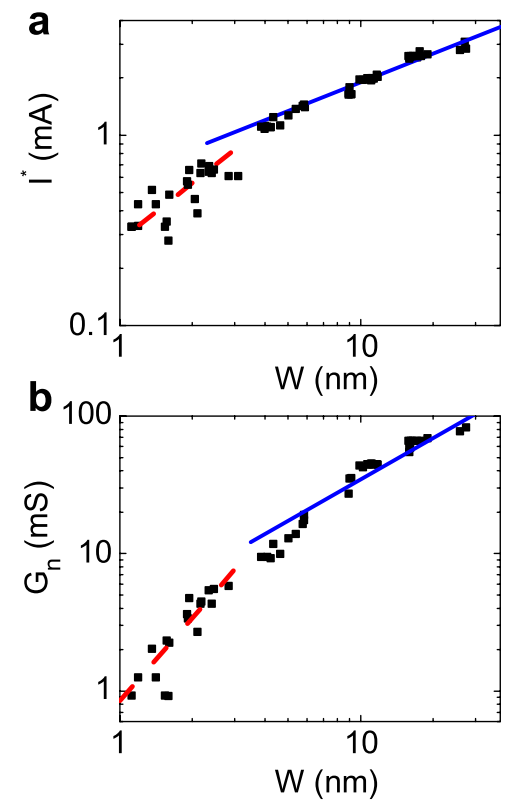

FIG. 4 (color online). Width dependence of $I^{*}$ and $G_{n}$. (a) $I^{*}$ is plotted against the corresponding neck width of the constrictions for the sample in Fig. 1. The solid (blue) line represents a powerlaw fit to $I^{*} \sim W^{1 / 2}$ and the dashed (red) line represents $I^{*} \sim W$. (b) $G_{n}$ plotted against neck width where the solid (blue) line represents a power-law fit to $G_{n} \sim W$ and the dashed (red) line represents $G_{n} \sim W^{2}$. 
case of transgranular electromigration at the sub- $20 \mathrm{~nm}$ size scale.

The above analysis indicates that the primary role of the current is to facilitate thermal excitation of atoms on the surface that are removed by current-induced forces. This is in contrast to the case of macroscopic polycrystalline leads where atoms migrate mostly along grain boundaries. In that case the primary role of the electric current is to generate a wind force that competes against the induced structural stresses of the wire, resulting in a minimum wire (or "Blech") length that can be electromigrated for a given uniform temperature and current [40]. Electromigration by the unzipping mechanism that we propose, which requires atomically smooth surfaces, also differs from electromigration in narrow $\mathrm{Al}$ and $\mathrm{Al}-\mathrm{Cu}$ interconnects where transgranular voids likely result from the catastrophic stress buildup due to the oxide surfaces [35,36].

These in situ TEM data reveal that nanoscale electrodes formed by FCE have a number of significant advantages for use as molecular-scale contacts. The thin and pointed geometry of the leads provides a well-defined, crystalline environment for molecular binding and significantly increased electrostatic coupling between a bound molecule and the backgate. Electrostatic simulations of this geometry indicate a roughly 2000 -fold increase in gate coupling compared to leads fabricated with standard nanolithographic techniques (see online supplementary information [15]). Unzipping is a unique mechanism for nanogap formation that could have significant advantages over other nanofabrication approaches since it only involves the movement of the least bound edge atoms while leaving the bulk of the leads intact. Finally, the crystal faceting and reversibility of the process unequivocally demonstrate that the electrodes are formed through a well-controlled electromigration process, as opposed to melting.

We acknowledge useful conversations with David P. Pope, Eugene J. Mele, and Douglas M. Yates. This work was supported by the Nano/Bio Interface Center through the National Science Foundation (NSF) under NSEC DMR-0425780, the NSF-NIRT Grant No. 0304531, the MRSEC Program of the NSF under Grant No. DMR0520020, and the Intelligence Community Postdoctoral program.

Note added. - While this manuscript was under review, related work was reported by Heersche et al. [41].

*drstrach@sas.upenn.edu †cjohnson@physics.upenn.edu

[1] H.S.J. van der Zant et al., Faraday Discuss. 131, 347 (2006).

[2] H. Park et al., Appl. Phys. Lett. 75, 301 (1999).

[3] G. Esen and M. S. Fuhrer, Appl. Phys. Lett. 87, 263101 (2005).

[4] M. L. Trouwborst, S. J.v.d. Molen, and B.J.v. Wees, J. Appl. Phys. 99, 114316 (2006).
[5] G. K. Ramachandran et al., Nanotechnology 16, 1294 (2005).

[6] D. R. Strachan et al., Appl. Phys. Lett. 86, 043109 (2005).

[7] A. A. Houck et al., Nano Lett. 5, 1685 (2005).

[8] V.C.-Y. Shih et al., Proceedings of the 12th International Conference on Solid-State Sensors, Actuators and Microsystems (Transducers '03), Boston, 2003 (IEEE, Piscataway, NJ, 2003), p. 1530.

[9] D. R. Strachan et al., Nano Lett. 6, 441 (2006).

[10] R. Sordan et al., Appl. Phys. Lett. 87, 013106 (2005).

[11] J. I. Gonzalez et al., Phys. Rev. Lett. 93, 147402 (2004).

[12] I. A. Blech and E. S. Meieran, Appl. Phys. Lett. 11, 263 (1967).

[13] I. A. Blech and E. S. Meieran, J. Appl. Phys. 40, 485 (1969).

[14] R. Rosenberg and L. Berenbaum, Appl. Phys. Lett. 12, 201 (1968).

[15] See EPAPS Document No. E-PRLTAO-100-009805 for five movie files and text discussing finite element simulations, unzipping, and atomic electromigration rates. For more information on EPAPS, see http://www.aip.org/ pubservs/epaps.html.

[16] P. J. Rous et al., J. Appl. Phys. 95, 2477 (2004).

[17] J. Dong and B. A. Parviz, Nanotechnology 17, 5124 (2006).

[18] H. Ishida, Phys. Rev. B 49, 14610 (1994).

[19] D. N. Bly and P. J. Rous, Phys. Rev. B 53, 13909 (1996).

[20] P. J. Rous, T. L. Einstein, and E. D. Williams, Surf. Sci. 315, L995 (1994).

[21] O. Bondarchuk et al., Phys. Rev. Lett. 99, 206801 (2007).

[22] P. J. Rous and D. N. Bly, Phys. Rev. B 62, 8478 (2000).

[23] K. O’Neill, E. A. Osorio, and H. S. J. van der Zant, Appl. Phys. Lett. 90, 133109 (2007).

[24] T. Taychatanapat et al., Nano Lett. 7, 652 (2007).

[25] D. E. Johnston, D. R. Strachan, and A. T. Johnson, Nano Lett. 7, 2774 (2007).

[26] A. Christou, Electromigration and Electronic Device Degradation (Wiley-Interscience, New York, 1994).

[27] M.F. Lambert et al., Nanotechnology 14, 772 (2003).

[28] M. Mahadevan and R. M. Bradley, Phys. Rev. B 59, 11037 (1999).

[29] J. Cho, M. R. Gungor, and D. Maroudas, Appl. Phys. Lett. 88, 221905 (2006).

[30] M. R. Gungor and D. Maroudas, Appl. Phys. Lett. 72, 3452 (1998).

[31] W. Wang, Z. Suo, and T.-H. Hao, J. Appl. Phys. 79, 2394 (1996).

[32] T. O. Ogurtania and A. Celik, J. Appl. Phys. 100, 043504 (2006).

[33] M. R. Gungor and D. Maroudas, J. Appl. Phys. 85, 2233 (1999).

[34] J. A. Prybyla et al., Appl. Phys. Lett. 73, 1083 (1998).

[35] J. H. Rose, Appl. Phys. Lett. 61, 2170 (1992).

[36] J. John E. Sanchez, O. Kraft, and E. Artz, Appl. Phys. Lett. 61, 3121 (1992).

[37] C. Durkan, M. A. Schneider, and M.E. Welland, J. Appl. Phys. 86, 1280 (1999).

[38] B.D. Knowlton, J.J. Clement, and C. V. Thompson, J. Appl. Phys. 81, 6073 (1997).

[39] D. Aherne, A. Satti, and D. Fitzmaurice, Nanotechnology 18, 125205 (2007).

[40] I. A. Blech, J. Appl. Phys. 47, 1203 (1976).

[41] H. B. Heersche et al., Appl. Phys. Lett. 91, 072107 (2007). 